\title{
VECTOR REPRESENTATION OF RESONATORS IN EIGENCOUPLING STATE SPACE AND EIGENLOSS STATE SPACE OF PIEZOELECTRIC CERAMICS
}

\author{
J. G. SMITS and A. J. MOUTHAAN
}

Dept. of Elect., Eng., Twente Univ. of Technology, Enschede, The Netherlands

\begin{abstract}
The calculation of the vector representation of resonators in the coupling and loss eigenstate spaces is presented. The coupling and loss eigenstates are located in a Cartesian coordinate system, together with the vector representation of resonators. The convenience of this representation is illustrated by calculations of the coupling factor and the loss factor in an octant of space in which resonators are positioned.
\end{abstract}

Nomenclature

$\left(c_{i j}^{D}\right), c^{D} \quad(i, j$ element of the $)$ matrix $c$, the elastic stiffness matrix at constant dielectric displacement.

$\left(d_{i j}\right), \boldsymbol{d} \quad(i, j$ element of the) matrix $d$, piezoelectric strain coefficient.

D dielectric displacement vector.

$e_{i j} \quad i, j$ element of the matrix $e$, piezoelectric stress coefficient.

F 9 dimensional state vector, containing 6 elastic strain elements $T_{i}, i=$ $1, \ldots, 6$ and 3 electric fields $E_{i}, j=1,2,3$.

$\mathbf{F}_{m} \quad$ state vector which is also a median vector

$g_{i j} \quad i, j$ element of the matrix $g$, the inverse piezoelectric stress coefficient.

$h_{i j} \quad i, j$ element of the matrix $h$, the inverse piezoelectric strain coefficient.

$k$ coupling factor.

$\mathbf{M}_{0} \quad$ the elastic-dielectric-piezoelectric matrix

$$
\left[\begin{array}{ll}
s^{E} & d^{t} \\
d & \varepsilon^{T}
\end{array}\right]
$$

in which the elements $d$ are made equal to zero.

$\mathbf{M}_{1}$ the elastic-dielectric-piezoelectric matrix

$$
\left[\begin{array}{ll}
s^{E} & d^{t} \\
d & \varepsilon^{T}
\end{array}\right]
$$

in which the elements $s^{E}$ and $\varepsilon^{T}$ are made equal to zero.

$\left(s_{i j}^{\mathrm{E}}\right), s^{\mathrm{E}} \quad(i, j$ element of the $)$ elastic compliance matrix $s$, at constant electric field E.

$\left(s_{i j}^{D}\right), s^{D} \quad(i, j$ element of the $)$ elastic compliance matrix $s$, at constant dielectric displacement $D$.

S elastic strain vector.

T elastic stress vector. 


$\begin{array}{ll}U & \text { internal energy. } \\ U_{E}, U_{\mathrm{M}}, U_{D} & \text { elastic, mutual, dielectric contribution to the internal energy. } \\ \left(\beta_{i j}^{\mathrm{T}}\right), \boldsymbol{\beta}^{\mathrm{T}} & (i, j \text { element of the impermeability matrix } \beta, \text { at constant stress. } \\ \boldsymbol{\beta}^{\mathrm{S}} & \text { impermeability matrix at constant strain. } \\ \left(\varepsilon_{i j}^{T}\right), \boldsymbol{\varepsilon}^{T} & (i, j \text { element of the) dielectric coefficient matrix at constant stress } T . \\ \omega & \text { angular frequency. }\end{array}$

\section{Introduction}

The theory of coupling and loss eigenstate has three uses:

A. It may be applied for characterization purposes, whereby a quick glance is sufficient to determine whether or not a material is suited for a special application.

B. Resonator vectors may be decomposed into the coupling and loss eigenstates, which enables us to conclude immediately which geometry of a (possibly oblique poled) sample might best serve our purpose.

C. It gives the extreme potentials of a material, and is here applied to both coupling and loss.

In recent years, a large number of new ceramic compositions have been investigated and tabulated in such well-known handbooks as Landolt-Börnstein [1] but also in more specialized books such as [2], [3] and [4]. When a designer wishes to construct a piezoelectric device, he may consult these tables and try to select a particular suitable material for his requirements. Usually he is inconvenienced by the fact that not all material constants of a certain composition are known. Moreover, the imaginary parts of the elastic, dielectric and piezoelectric constants are usually unknown, or they are measured at a frequency that differs greatly from the operating frequency of his own device $(\tan \delta$ of the dielectric constant is often measured at $1000 \mathrm{~Hz}$ ).

On the other hand, he may have some materials available, none of which really fulfill the requirements he imposes. In this case a choice of a particular device geometry and a certain poling axis, might serve him well, although the other extreme, where no device geometry or direction of excitation might be satisfactory, may also be true.

It is the purpose of this paper to present the calculations for this type of problem and to clarify the meaning of the coupling and loss eigenstate spaces. Further, it is explained why some geometries are more appropriate for certain applications than others. 
The concepts of eigencoupling and loss states were published in 1969 by Holland and EerNisse [5], but were mostly considered by the authors [6] to be a mathematical curiosity without technical applications. To the knowledge of the present authors it is the first time that practical situations are described in the framework of the eigenstate theory. Firstly we shall present the results of the calculations of the eigencoupling factor and the associated eigenstates. Further, the characteristic vector of a certain resonator geometry will be calculated and positioned in the eigencoupling space. This is done for thin rings, bars with parallel and perpendicular poling axis, radial resonators and finally for thickness dilation resonators.

The fourth section treats the renormalized coupling factor and shows how the eigencoupling space and the eigenloss space can be shifted into each other.

\section{Eigen or stationary coupling factors}

If a material is considered to be lossless, we can derive for the electroelastic contribution to the internal energy density:

$$
U=\frac{1}{2} \mathbf{T}^{t} \cdot \mathbf{S}+\frac{1}{2} \mathbf{D}^{t} \cdot \mathbf{E}
$$

or, using the constituent equations, of piezoelectricity [11]

$$
\begin{aligned}
U & ={ }_{2}^{1} \mathbf{T}^{t} \cdot \boldsymbol{s}^{E} \cdot \mathbf{T}+\frac{1}{2} \mathbf{T}^{t} \cdot \boldsymbol{d}^{\mathrm{t}} \cdot \mathbf{E}+\frac{1}{2} \mathbf{E}^{t} \cdot \boldsymbol{d} \cdot \mathbf{T}+\frac{1}{2} \mathbf{E}^{\mathrm{t}} \cdot \boldsymbol{\varepsilon}^{\mathrm{T}} \cdot \mathbf{E} \\
& =U_{E}+2 U_{M}+U_{D} .
\end{aligned}
$$

We define the coupling factor as:

$$
k=\frac{2 U_{M}}{U_{E}+U_{\mathbf{D}}} .
$$

The stationary values of $k$ are found as solutions of:

$$
\frac{\partial k}{\partial T_{i}}=0, \quad \frac{\partial k}{\partial E_{i}}=0 \quad i=1, \ldots, 6 ; \quad j=1, \ldots, 3 .
$$

\section{Representation of resonators in the coupling state space}

It appeared that we could define a particular vector having useful properties. It is called a median vector $F_{m}$ and it is defined by the 
following properties $P$ :

$P_{\mathrm{i}}$ a median vector is normalized to have dielectric plus elastic energy density equal to $1 \mathrm{~J} / \mathrm{m}^{3}$

$U_{E}+U_{D}=1 \mathrm{~J} / \mathrm{m}^{3}$

$P_{\mathrm{ii}}$ the distribution of energy is such that

$U_{E}=U_{D}=\frac{1}{2} \mathrm{~J} / \mathrm{m}^{3}$.

If the coupling factor $k$ associated with a vector $F$ is written as $k(F)$, it readily follows from these properties that:

$P_{\mathrm{iii}}$ when the length in $\left(T_{1}, T_{2}, T_{3}\right)$ space of a median vector $F_{m}$ is changed, and at the same time the electric field is also changed then the new vector $F_{m}+\Delta F$ may still have property $P_{i}$, but no longer $P_{i i}$, and further the following relations are valid

$$
\begin{array}{lll}
k\left(F_{m}+\Delta F\right)<k\left(F_{M}\right) & \text { if } & \mathbf{F}_{m}^{t} \cdot \boldsymbol{M}_{0} \cdot \mathbf{F}_{1}>0, \\
k\left(F_{m}+\Delta F\right) \geqslant k\left(F_{m}\right) & \text { if } & \mathbf{F}_{m}^{t} \cdot \boldsymbol{M}_{0} \cdot \mathbf{F}_{1} \leqslant 0 .
\end{array}
$$

$P_{\mathrm{iv}}$ when a median vector $F_{m}$ is infinitesimally changed to a new median vector $\mathbf{F}_{m}^{\prime}=\mathbf{F}_{m}+\Delta \mathbf{F}$, then

$$
\begin{array}{lll}
k\left(F_{m}+\Delta F_{m}\right)>k\left(F_{m}\right) & \text { if } \quad \Delta \mathbf{F}_{m}^{t} \cdot \boldsymbol{M}_{0} \cdot \mathbf{F}_{1}>0, \\
k\left(F_{m}+\Delta F_{m}\right) \leqslant k\left(F_{m}\right) & \text { if } \quad \Delta \mathbf{F}_{m} \cdot \boldsymbol{M}_{0} \cdot \mathbf{F}_{1} \leqslant 0 .
\end{array}
$$

The proof of $P_{\mathrm{iii}}$ and $\boldsymbol{P}_{\mathrm{iv}}$ is simple and will not be presented here. Note that all coupling eigenstates are median vectors.

For ease of reference the coupling eigenstates are tabulated in table 1. It is clear that we can unambiguously distinguish shear modes from dilatation modes, because shear modes are not piezoelectrically coupled by fields parallel to the spontaneous polarization. Only fields perpendicular to this axis excite shear deformations. This is reflected by the field of zero's in the matrix of the constituent relations, above, below and to the right and left of the inner shear heart of the matrix [11]. In figure 1 the eigenstates are drawn, together with their coupling factors. The $x$ and $y$-axes point out of the paper, at angles of $45^{\circ}$, allowing us to draw $\mathbf{F}_{1}, \mathbf{F}_{2}$ and $\mathbf{F}_{3}$ in the plane of the paper, while $\mathbf{F}_{9}$ points towards the reader. In the analytical expression for $\mathbf{F}_{9}$ we find no dependence on piezoelectric coefficients; apparently this mode has no relation to piezoelectricity. It represents elastic forces in the $+x$ and $-y$ direction, and it is therefore equivalent to the Lamé mode in 
TABLE 1. Eigenstates and eigencoupling factors of poled ferroelastic ceramics.

\begin{tabular}{|c|c|c|c|c|c|c|c|c|}
\hline$F_{1}$ & $F_{2}$ & $F_{3}$ & $F_{4}$ & $F_{5}$ & $F_{6}$ & $F_{7}$ & $F_{8}$ & $F_{9}$ \\
\hline$\frac{e_{31}}{\sqrt{\varepsilon_{33}^{T}-\varepsilon_{33}^{\mathrm{s}}}}$ & $\frac{e_{31}}{\sqrt{\varepsilon_{33}^{\mathrm{T}}-\varepsilon_{33}^{\mathrm{s}}}}$ & $\frac{\alpha}{2}$ & 0 & 0 & 0 & 0 & 0 & $\frac{1}{\sqrt{s_{66}}}$ \\
\hline$\frac{e_{31}}{\sqrt{\varepsilon_{33}^{T}-\varepsilon_{33}^{\mathrm{S}}}}$ & $\frac{e_{31}}{\sqrt{\varepsilon_{33}^{T}-\varepsilon_{33}^{s}}}$ & $\frac{\alpha}{2}$ & 0 & 0 & 0 & 0 & 0 & $\frac{-1}{\sqrt{s_{66}}}$ \\
\hline$\frac{e_{31}}{\sqrt{\varepsilon_{33}^{T}-\varepsilon_{33}^{s}}}$ & $\frac{e_{33}}{\sqrt{\varepsilon_{33}^{\mathrm{T}}-\varepsilon_{33}^{\mathrm{s}}}}$ & $\frac{-d_{31} \alpha}{d_{33}}$ & 0 & 0 & 0 & 0 & 0 & 0 \\
\hline 0 & 0 & 0 & $\frac{1}{\sqrt{s_{55}}}$ & 0 & 0 & 0 & $\frac{1}{\sqrt{s_{55}}}$ & 0 \\
\hline 0 & 0 & 0 & 0 & $\frac{1}{\sqrt{s_{55}}}$ & 0 & $\frac{1}{\sqrt{s_{55}}}$ & 0 & 0 \\
\hline 0 & 0 & 0 & 0 & 0 & $\frac{1}{\sqrt{s_{66}}}$ & 0 & 0 & 0 \\
\hline 0 & 0 & 0 & 0 & $\frac{-1}{\sqrt{\varepsilon_{11}^{T}}}$ & $\frac{1}{\sqrt{\varepsilon_{11}^{\mathrm{T}}}}$ & $\frac{1}{\sqrt{\varepsilon_{11}^{T}}}$ & 0 & 0 \\
\hline 0 & 0 & 0 & $\frac{-1}{\sqrt{\varepsilon_{11}^{T}}}$ & 0 & 0 & 0 & $\frac{1}{\sqrt{\varepsilon_{11}^{T}}}$ & 0 \\
\hline$\frac{1}{\sqrt{\varepsilon_{33}^{T}}}$ & $\frac{-1}{\sqrt{\varepsilon_{33}^{T}}}$ & $\frac{1}{\sqrt{\varepsilon_{33}^{T}}}$ & 0 & 0 & 0 & 0 & 0 & $\frac{1}{\sqrt{\varepsilon_{33}^{T}}}$ \\
\hline$k_{1}$ & $k_{2}$ & $k_{3}$ & $k_{4}$ & $k_{5}$ & $k_{6}$ & $k_{7}$ & $k_{8}$ & $k_{9}$ \\
\hline 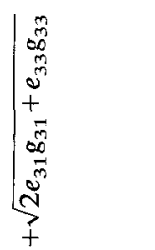 & 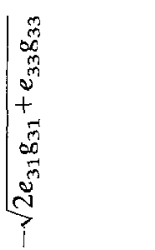 & 0 & 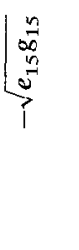 & 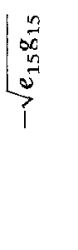 & 0 & 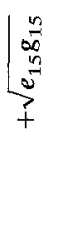 & $\begin{array}{l}\mid \begin{array}{l}n \\
0 \\
n \\
n \\
z \\
+\end{array} \\
+\end{array}$ & 0 \\
\hline
\end{tabular}

The columns form the eigenstates, while their associated eigencoupling factors are printed vertically below them. The nonzero elements of $F_{4}$ to $F_{9}$ are the inner shear heart.

plates, which cannot be excited in ceramics [7]. In figure 2 half of the elastic part of the coupling space of PZT4 is shown [8]. A net formed by solid lines of constant $(\theta, \phi)$ (in polar coordinates) indicates the shape of a three-dimensional ellipsoid. The length of a vector from the origin to the surface of this ellipsoid indicates the required elastic force in the $(\theta, \phi)$ direction necessary to obtain an elastic energy density of $\frac{1}{2} \mathrm{~J} / \mathrm{m}^{3}$ in the material. 


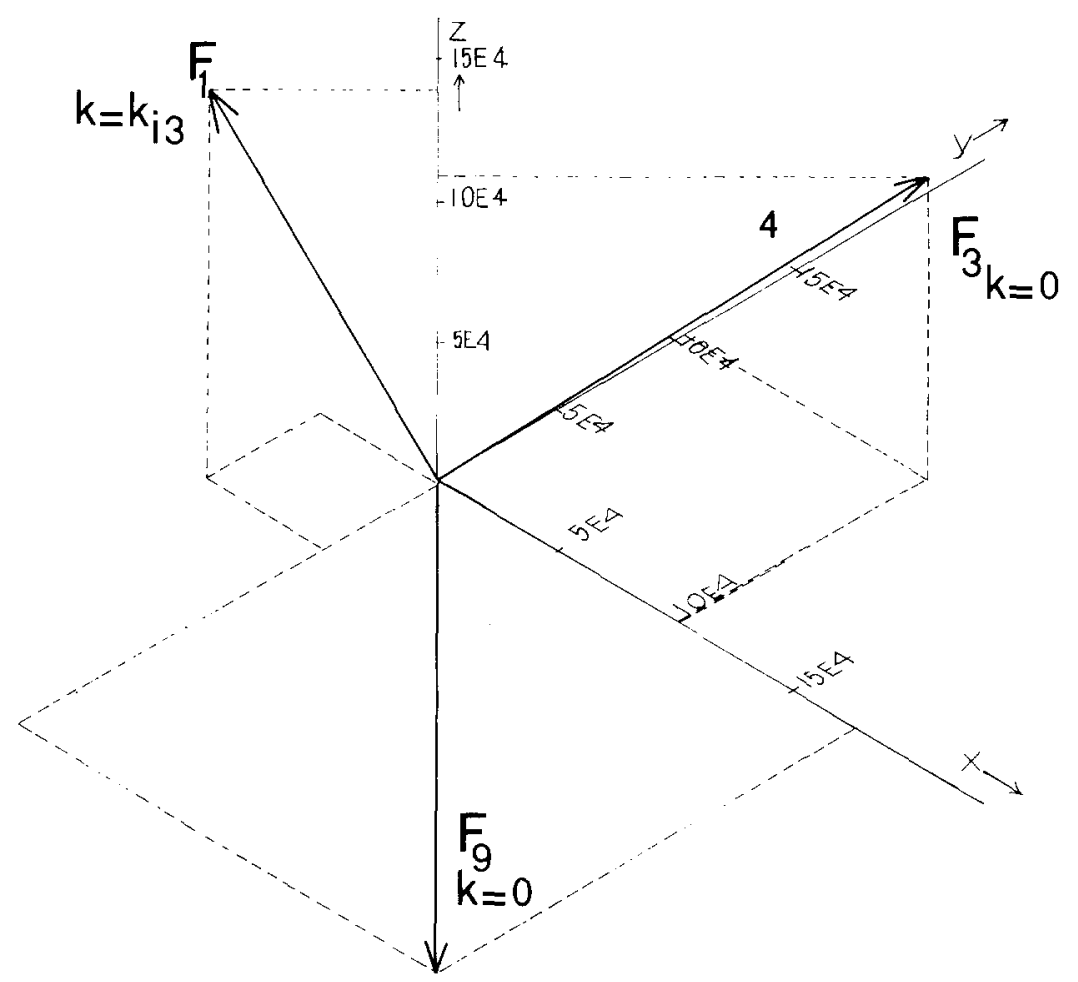

Fig. 1 Piezoelectric eigenstates of a ferroelectric ceramic.

The eigenstate $F_{9}$ is perpendicular to the plane of the paper. This picture is drawn by means of a computer program, which calculated the location of the projection of a point on the surface onto a plane, spanned by the vectors $(0,0,1)$ and $(1,1,0)$. The ellipsoid is drawn as it is percepted by an observer, whose eye is at a distance from the plane of the paper, which is approximately equal to three times the length of the major axis of the ellipsoid.

The contours in broken lines running from the lower left to the upper right, are the lines of constant values of the coupling factor. A normalized vector with angles $(\theta, \phi)$ in polar coordinates, has a coupling factor indicated by the value of $k$ at the point $(\theta, \phi)$ on the surface of the ellipsoid. We see that a vector of normalized length and of appropriate dielectric energy content in the direction of the $x$-axis, will reach a coupling factor $k_{x}=-0.32$, while a vector along the $z$-axis will achieve $k_{z}=0.68$. When the vector of a particular state does not 


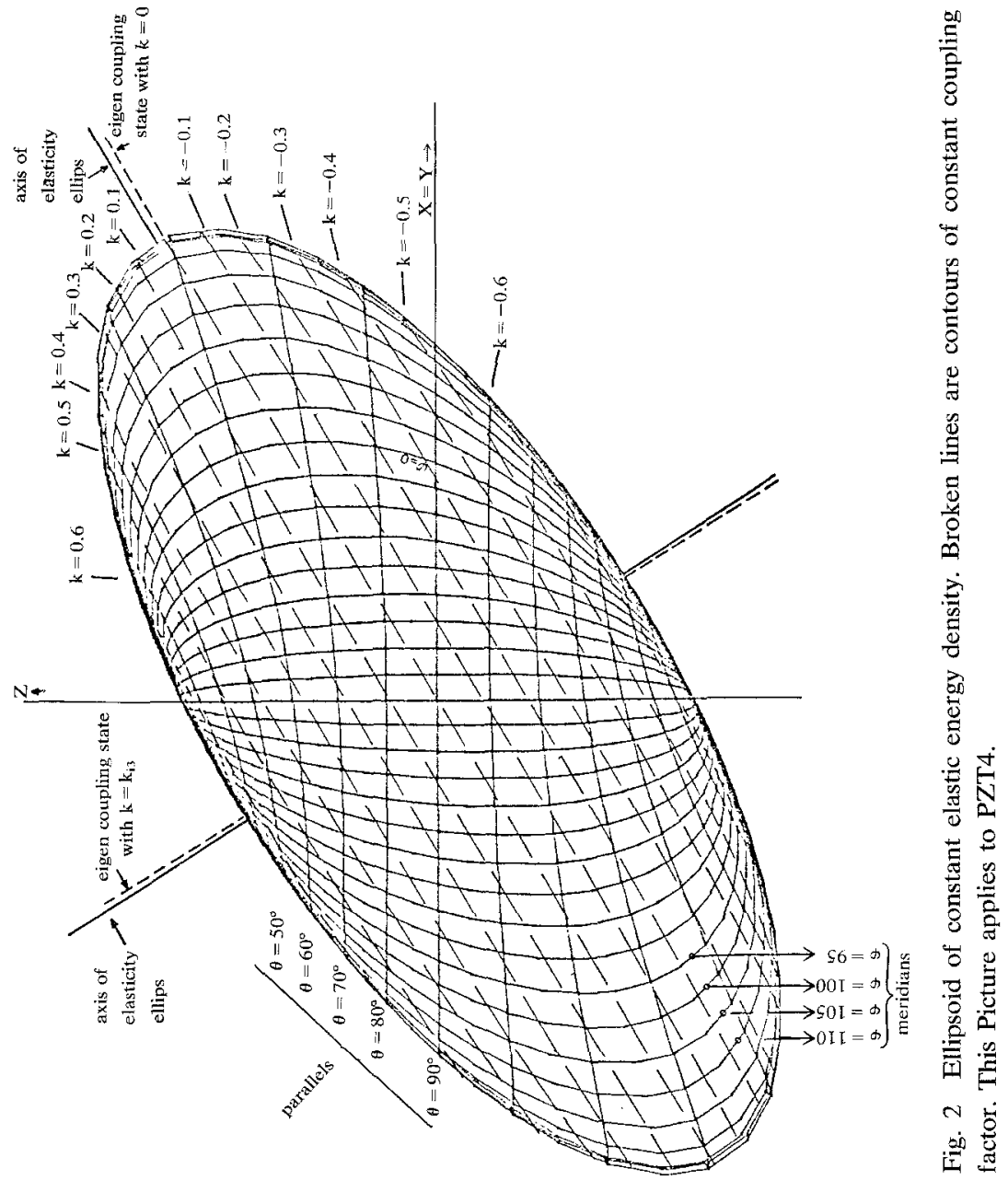


contain an appropriate amount of dielectric energy (it is not a median vector), the modulus of the coupling factor is always lower. The limiting vectors with elastic or dielectric energy content equal to zero, are associated with coupling factors zero.

It should be noted, that in the calculations of the eigencoupling states, these states are without phase components, which excludes phenomena very close to elastic resonance. At this point it is important to distinguish the purely elastical phenomenon of resonance, causing phase shifts between $T$ and $S$, and the phenomenon of coupling between the dielectric and elastic properties. For resonators it is clear that good coupling properties will result in large phase variations near resonance, and a large frequency interval between the so-called resonance frequency and the antiresonance frequency. This means, that even if we exclude phase shifting in the eigenspace, the vector representation bears information about the relative quality of resonators.

We are now in a position to start calculations of the state vector of various modes. Because of the lack of spatial variations of the elastic forces, we begin with the radial mode in samples with the shape of a thin ring.

\section{A. RADIAL MODE IN THIN RINGS}

All volume elements are subjected to the same forces $T_{1}$ and $E_{3}$, whether the electrodes are inside and outside the ring or at the top and bottom plane [8]. The constituent relations for this set of boundary conditions reduce to:

$$
\begin{aligned}
& S_{1}=s_{11}^{E} T_{1}+d_{31} E_{3}, \\
& D_{3}=d_{31} T_{1}+\varepsilon_{33}^{T} E_{3} . \\
& T_{1}=\frac{d_{31}}{s_{11}^{E}}\left(\frac{\omega^{2}}{\omega_{0}-\omega^{2}}\right) E_{3}=\frac{d_{31}}{s_{11}^{E}} E_{3} R,
\end{aligned}
$$

where

$$
\begin{aligned}
\omega_{0}^{2} & =1 / a^{2} \rho s_{11}^{E} \\
a & =\text { radius of the ring }
\end{aligned}
$$

in which $R$ is the resonance term, and in which the time dependence $\mathrm{e}^{\mathrm{i} \omega t}$ is suppressed. 
According to the definition of $k$ in equation (3) we find:

$$
k=\frac{2 U_{M}}{U_{E}+U_{D}}=\frac{d_{31} T_{1} E_{3}}{\frac{1}{2} s_{11}^{E} T_{1}^{2}+\frac{1}{2} \varepsilon_{33}^{T} E_{3}^{2}} .
$$

The electric field $E_{3}$ is adjusted in such a way that:

$$
\frac{1}{2} s_{11}^{E} T_{1}^{2}+\frac{1}{2} \varepsilon_{33}^{T} E_{3}^{2}=1 \mathrm{~J} / \mathrm{m}^{3}
$$

which yields:

$$
\begin{aligned}
& E_{3}=\left(\frac{1}{2} \varepsilon_{33}^{T}+\frac{1}{2} \frac{d_{31}^{2}}{s_{11}^{E}} R^{2}\right)^{-1 / 2}, \\
& T_{1}=\frac{d_{31}}{s_{11}^{E}} R\left(\frac{1}{2} \varepsilon_{33}^{T}+\frac{1}{2} \frac{d_{31}^{2}}{s_{11}^{E}}\right)^{-1 / 2} .
\end{aligned}
$$

By substitution of (14) and (15) in (12) we now find for $k$ :

$$
k=\frac{d_{31}^{2}}{s_{11}^{E}} \frac{R}{\left(\frac{1}{2} \varepsilon_{33}^{T}+\frac{d_{31}^{2}}{2 s_{11}^{E}} R^{2}\right)} .
$$

The frequencies of the extreme values for $k$ are found to be:

$$
\omega_{1,2}^{2}\left(k=k_{\text {extr }}\right)=\frac{\omega_{0}^{2}}{1 \pm \frac{d_{31}}{\sqrt{s_{11}^{E} \varepsilon_{33}^{T}}}},
$$

where the plus and the minus signs correspond to a maximum and a minimum for $k$ of:

$$
k_{ \pm}=\frac{ \pm d_{31}}{\sqrt{s_{11}^{E} \varepsilon_{33}^{T}}} \text {. }
$$

Examination of $T_{1}, E_{3}$ and the energy densities $U_{E}$ and $E_{D}$ shows, that at these frequencies

$$
T_{1}=\frac{ \pm 1}{\sqrt{s_{11}^{E}}} \quad E_{3}=\frac{1}{\sqrt{\varepsilon_{33}^{T}}}
$$

and

$$
U_{E}=U_{D}=\frac{1}{2}
$$

so we conclude that the state vector of the ring resonator is a median vector at these frequencies. Below and above these frequencies the 
behavior of $k$ is reflected by $P_{\mathrm{iii}} \cdot T_{1}$ is the only component in a ring, and consequently, the elastic part of the vector representing of the ring (the state vector), is collinear with the $x$-axis. The vector, which increases quadratically at low frequencies, contacts the surface of the ellipsoid at a frequency $\omega_{1}$. (See equation (17)), where the coupling factor is a maximum. It increases asymptotically to resonance, where sign reversal occurs and now decreasing with increasing frequency, it again contacts the surface of the ellipsoid at $\omega=\omega_{2}$. This point is at the opposite side of the ellipsoid where the coupling factor equals $-d_{31} / \sqrt{s_{11}^{E} \varepsilon_{33}^{T}}$.

\section{B. OTHER MODES OF VIBRATION}

Before we proceed, we realize that in bars and plates $\mathbf{T}$ and $\mathbf{E}$ depend on the place. At any place $\mathbf{r}$ in the body of a resonator, we may consider an infinitesimal volume element $\mathrm{d} x d y \mathrm{~d} z=\mathrm{d} r$ in which we assume $\mathbf{T}(\mathbf{r})$ and $\mathbf{E}(\mathbf{r})$ to be constant. Then we can write

$$
u(\mathbf{r})=\frac{1}{2} \mathbf{S}^{\mathbf{t}}(\mathbf{r}) \cdot \mathbf{T}(\mathbf{r})+\frac{1}{2} \mathbf{D}^{t}(\mathbf{r}) \cdot \mathbf{E}(\mathbf{r}) .
$$

In order to facilitate the calculations, we collect the six elastic forces and three electric fields in a generalized force vector $\mathbf{F}$ of nine elements, we designate the elastic and dielectric matrices by $M_{0}$ and the piezoelectric matrix by $M_{1}$ according to

$$
\begin{aligned}
& M_{0}=\left(\begin{array}{cc}
s^{E} & 0 \\
0 & \varepsilon^{T}
\end{array}\right) \\
& M_{1}=\left(\begin{array}{ll}
0 & d^{t} \\
d & 0
\end{array}\right)
\end{aligned}
$$

and rewrite (20) as

$$
u(\mathbf{r})=\frac{1}{2} \mathbf{F}^{t}(\mathbf{r}) \cdot \boldsymbol{M}_{0} \cdot \mathbf{F}(\mathbf{r})+\frac{1}{2} \mathbf{F}^{t}(\mathbf{r}) \cdot \boldsymbol{M}_{1} \cdot \mathbf{F}(\mathbf{r}) .
$$

in which $u(\mathbf{r})$ is the local energy density. From (22) we define the local coupling factor $k(\mathbf{r})$ as

$$
k(\mathbf{r})=\frac{\frac{1}{2} \mathbf{F}^{t}(\mathbf{r}) \cdot \mathbf{M}_{1} \cdot \mathbf{F}(\mathbf{r})}{{ }_{2}^{1} \mathbf{F}^{t}(\mathbf{r}) \cdot \mathbf{M}_{0} \cdot \mathbf{F}(\mathbf{r})} .
$$


The total electroelastic energy is then:

$$
U=\int_{V} u(\mathbf{r}) \mathrm{d} r
$$

and we can introduce the "total coupling factor" $k_{V}$ as

$$
k_{V}=\frac{1}{V} \int_{V} k(\mathbf{r}) \mathrm{d} r
$$

where $V$ denotes the volume of the body.

\section{LENGTH EXPANDER BAR WITH FIELD PERPENDICULAR TO LENGTH}

The boundary condition for these resonators are the same as for the ring resonator, but Newton's equation has a different form, due to the spatial variation of $T[8]$. This leads to:

$$
T_{1}=\frac{d_{31}}{s_{11}^{\mathrm{E}}} E_{3}\left(\frac{\cos a x^{\prime}}{\cos a / 2}-1\right)=\frac{d_{31} E_{3}}{s_{11}^{\mathrm{E}}}\left(R_{\mathrm{s}}-1\right)
$$

in which $a=\omega l / v$, ( $v$ is velocity of sound propagation) and $x^{\prime}=\frac{1}{2}-x / l$. For the meaning of $x$ and $l$ we refer to figure 3 .

A plot of $T_{1}$ is shown in figure 4 , where the electric field $E_{3}$ is assumed to be $1 \mathrm{~V} / \mathrm{m}$. Here we see a $y$-axis which serves as the $T_{1}$ axis and an $x$-axis on which a point indicates the place in the bar at which the forces, energy densities and the coupling factor are evaluated. The free ends of the bar are equivalent to $x^{\prime}= \pm 0.5$, while the center of the bar corresponds to $x^{\prime}=0$. The reduced frequency axis, i.e. the $a$-axis which is perpendicular to $x$ and $y$-axes is shown pointing away from
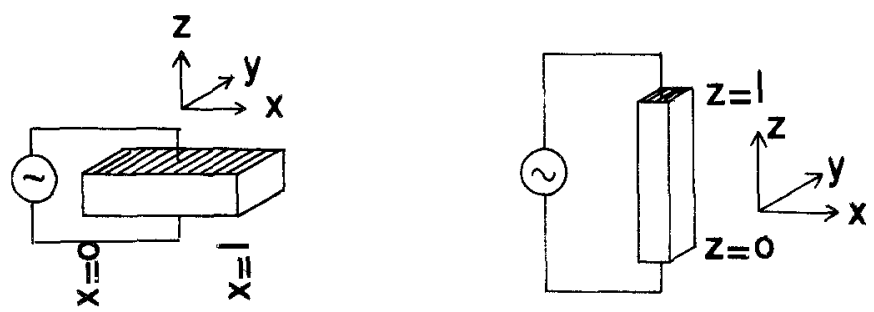

Fig. 3 The length expander bars with electric fields perpendicular and parallel to lengths. 


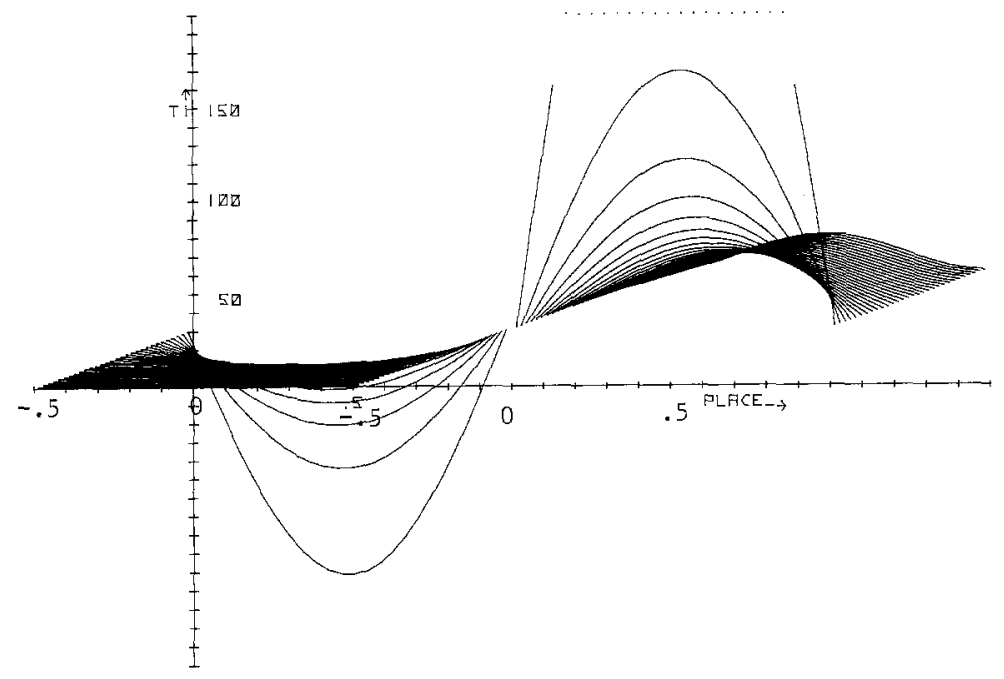

Fig. 4 Plot of $T_{1}$ (freq, place) perpendicular poled bar resonator.

the reader and slanting upwards. The dependence of $T_{1}$ on $x$ is calculated for different values of the frequency with increments of $\Delta a=0.05$. Resonance is then found at $a=\pi / 2$. For low frequencies the dependence of $T_{1}$ on $x$ is shown as a part of a negative cosine, while with increasing frequency $T_{1}$ extends in negative direction, changing its sign at resonance and again decreasing with increasing frequency above resonance. As the frequency dependence is calculated from d.c. to twice the resonance frequency, the diagram is shifted to the right above resonance, to avoid too many crossing lines confusing the reader. At $\omega=2 \omega_{\text {res }}$ the value of $T_{1}$ has not diminished, although the electrical admittance of the bar shows no particular behavior at this frequency. The local coupling factor is found by substituting (26) into (23), giving:

$$
k_{\mathrm{loc}}=\frac{d_{31}^{2}\left(R_{\mathrm{s}}-1\right) / s_{11}^{E}}{\frac{1}{2} s_{11}^{E}\left(\frac{d_{31}}{s_{11}^{E}}\left(R_{\mathrm{s}}-1\right)\right)^{2}+\frac{1}{2} \varepsilon_{33}^{\mathrm{T}}}=\frac{2 \kappa_{31}^{2}\left(R_{\mathrm{s}}-1\right)}{1+\kappa_{31}^{2}\left(R_{\mathrm{s}}-1\right)^{2}}
$$

in which the familiar cluster $d_{31}^{2} / \varepsilon_{33}^{T} s_{11}^{E}$ is denoted as $\kappa_{31}^{2}$. In figure 5 a plot is shown of the local coupling factor as a function of the position in the bar and of the frequency. The figure consists of two parts, below 
and above resonance. The part above resonance being again graphically shifted to the right, to avoid too many crossing lines. We see that at a frequency of $0.838 \times f_{\text {res }}$ [8] the local generalized force vector for the centre of the bar is a median vector. For frequencies closer to resonance, the local elastic energy density becomes too high, resulting in lower values of $k_{\mathrm{loc}}$. This causes of the maximum to be split into two maxima, which move outwards to the ends of the bar when the frequency approaches resonance. The definite integral of $k_{\mathrm{loc}}$ over the whole bar yields:

$$
\begin{aligned}
k_{\mathrm{tot}}= & \frac{4 \kappa_{31}^{2}(1+b)}{a b \beta}\left(p \cdot \log \left(\frac{\tan ^{2} \frac{a}{4}+2 r \tan \frac{a}{4}+s}{\tan ^{2} \frac{a}{4}-2 r \tan \frac{a}{4}+s}\right)\right. \\
& \left.+\frac{2(q-r p)}{\sqrt{s-r^{2}}}\left(\arctan \left(\frac{\tan \frac{a}{4}+r}{\sqrt{s-r^{2}}}\right)+\arctan \left(\frac{\tan \frac{a}{4}-r}{\sqrt{s-r^{2}}}\right)\right)\right),
\end{aligned}
$$

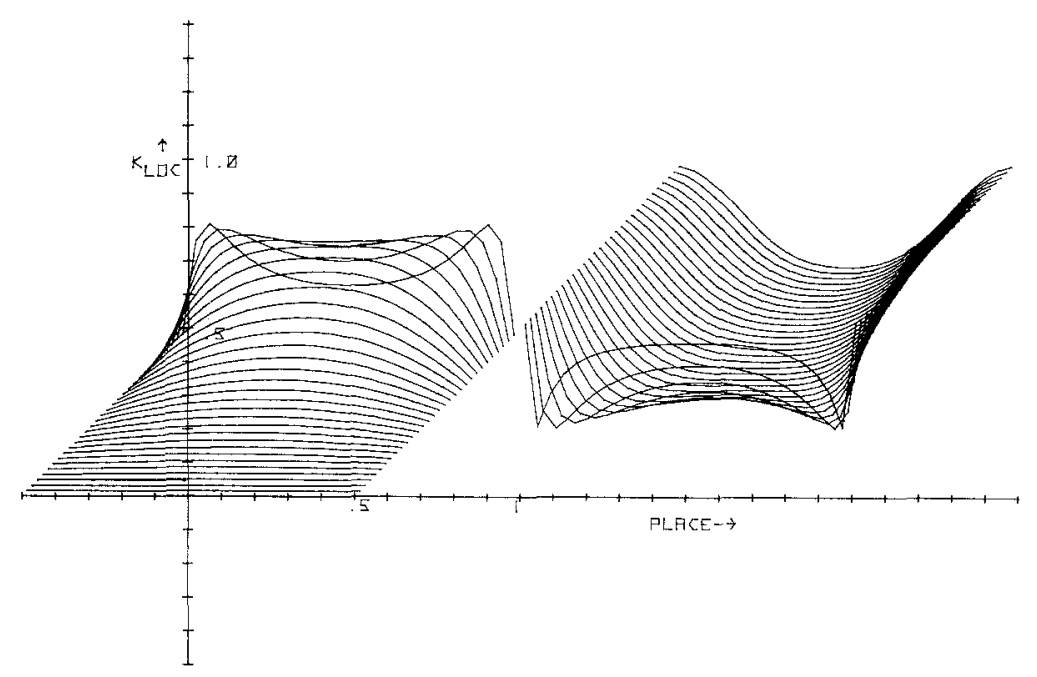

Fig. 5 Plot of a $k_{\text {loc }}$ (freq, place) perpendicular poled bar. 
where the following abbreviations are used:

$$
\begin{array}{ll}
a=\text { reduced frequency: } & \pi \omega / \omega_{0} \\
b=\cos a / 2 & \delta=(1-b) /(1+b) \\
\alpha=1+\kappa_{31}^{2}\left(1-1 / b^{2}\right) & s=\sqrt{\gamma / \beta} \\
\beta=1+\kappa_{31}^{2}(1+1 / b)^{2} & r=\sqrt{(s-\alpha / \beta) / 2} \\
\gamma=1+\kappa_{31}^{2}(1-1 / b)^{2} & p=(1+\delta / s) / 4 r .
\end{array}
$$

This expression is of considerable complexity, and for other geometries it becomes even more complicated, so no attempts were made to derive analytical expressions for the total coupling factor of the parallel poled bar and the plate resonator. In the coupling state space, each point in the bar has its own state vector, which is for all points collinear with the $x$-axis, but which has different magnitudes for different points. Of course, due to the symmetry of the bar with respect to the centre, symmetrically located points have the same state vector. These vectors contact the surface of the ellipsoid each at a different frequency depending on position, in the bar. At resonance they all change sign while with increasing frequency, they all pass the ellipsoid surface again. For a large portion of the bar the local state vectors remain very close to the surface of the ellipsoid, resulting in a coupling factor which is almost maximum, for this part as mentioned before.

\section{LENGTH EXPANDER BAR WITH FIELD PARALLEL TO LENGTH}

For this geometry (see figure 3 ) the boundary conditions are given by

$$
\begin{aligned}
& T_{1}=T_{2}=0, \\
& T_{3}(0,1)=0,
\end{aligned}
$$

while we apply a voltage difference $\phi \mathrm{e}^{j \omega t}$ to the electrodes. From Maxwell's equation $\boldsymbol{\nabla} \cdot \mathbf{D}=\rho$ follows for an uncharged insulating medium as an electrical boundary condition:

$$
\frac{\partial D_{3}}{\partial x_{3}}=0 .
$$

It is therefore useful to consider as the appropriate set of constituent relations:

$$
\begin{aligned}
& S_{3}=s_{33}^{D} T_{3}+g_{33} D_{3}, \\
& E_{3}=-g_{33} T_{3}+\beta_{33}^{T} D_{3} .
\end{aligned}
$$


Newton's equation for this type of excitation gives:

$$
\rho \frac{\partial^{2} \xi_{3}}{\partial t^{2}}=\frac{1}{s_{33}^{D}} \frac{\partial^{2} \xi_{3}}{\partial x_{3}^{2}}
$$

from which follows

$$
S_{3}=g_{33} D_{3} \frac{\cos a x_{3}}{\cos a / 2}=g_{33} D_{3} R_{B}
$$

where $\xi$ is the displacement of a particle from its equilibrium position, and $a$ is again the reduced frequency defined by (29). Substitution in (32) yields:

$$
\begin{aligned}
& T_{3}=\frac{g_{33}}{s_{33}^{D}} D_{3}\left(R_{\mathrm{B}}-1\right), \\
& E_{3}=\left(\beta_{33}^{T}+\frac{g_{33}^{2}}{s_{33}^{D}}\left(1-R_{\mathrm{B}}\right)\right) D_{3} .
\end{aligned}
$$

In order to evaluate our electrical boundary condition (31) we integrate the electric field across the bar, because as

$$
E_{3}=-\frac{\partial \phi}{\partial x_{3}}, \text { it follows that } \phi=-\int_{-1 / 2}^{1 / 2} E_{3} \mathrm{~d} x_{3} .
$$

This can be used to calibrate $E_{3}$, and we find this as a function of $\phi$ given by

where

$$
E_{3}=\left(1-R_{\mathrm{B}} \kappa_{33}^{2}\right) \frac{\phi}{-1+\kappa_{33}^{2} \frac{\tan a / 2}{a / 2}},
$$

$$
\kappa_{33}^{2}=\frac{d_{33}^{2}}{s_{33}^{E} \varepsilon_{33}^{T}}=\frac{g_{33}^{2}}{s_{33}^{E} \beta_{33}^{T}}
$$

and we calculate $T_{3}$ as a function of $\phi$ by means of equations (35) and (36):

$$
T_{3}=\frac{d_{33}}{s_{33}^{E}}\left(R_{B}-1\right) \frac{\phi}{-1+\kappa_{33}^{2} \frac{\tan a / 2}{a / 2}}
$$

Equations (38) and (40) express $E_{3}$ and $T_{3}$ as functions of the external voltage difference $\phi$ and of the frequency $\omega$ (by means of the resonance term $R_{B}$ (see (34))). If we maintain the voltage amplitude $\phi$ at a 
constant level $(\phi=1)$, we can calculate and plot $T_{3}$ and $E_{3}$ versus $\omega$ : these plots are shown in figures 6 and 7. The plots are not trivial variations of the plot of $T_{1}$ for the perpendicular poled bar, because they show the phenomenon of the so-called "precursory" resonance. While the "true" resonance frequency is found as the resonance of $S_{3}$, we observe a shifting to lower frequencies of the resonances of $T_{3}$ and $E_{3}$ which is caused by the occurrence of a zero in the numerator of (38) and (40) for a frequency lower than resonance. The precursory resonance frequency $a_{p}$ is found as a solution of a transcendental equation

$$
k_{33}^{2}=\frac{\tan a_{\mathrm{p}} / 2}{a_{\mathrm{p}} / 2}
$$

which shows that the shifting is larger for greater piezoelectric effects. A similar shifting was already observed in piezoelectric plates by Tiersten [9]. At the "true" resonance, the pole in the denominator is cancelled by the pole in the numerator, and no discontinuity is observed in the behavior of $T_{3}$ and $E_{3}$. In our example we used $\kappa_{33}=0.7$, which gives a precursory resonance frequency of

$$
0.748 f_{\text {res }}, \quad \text { or } a_{p}=2.35 \text {. }
$$

Further, we observe that $E_{3}$ has negative values near precursory resonance, which indicates that an inverted potential difference exists

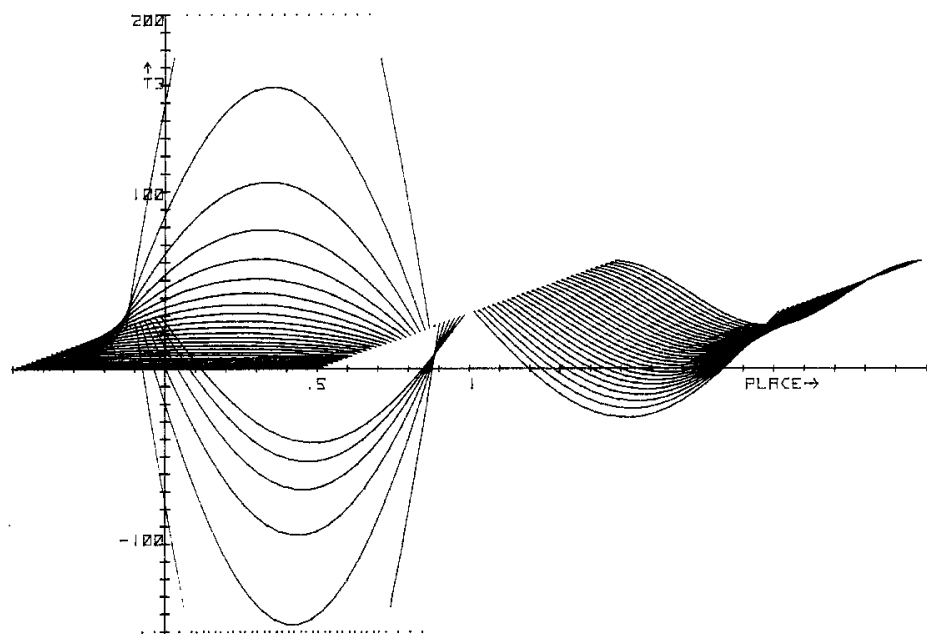

Fig. 6 Plot of $T_{3}$ (freq, place) parallel poled bar resonator. 


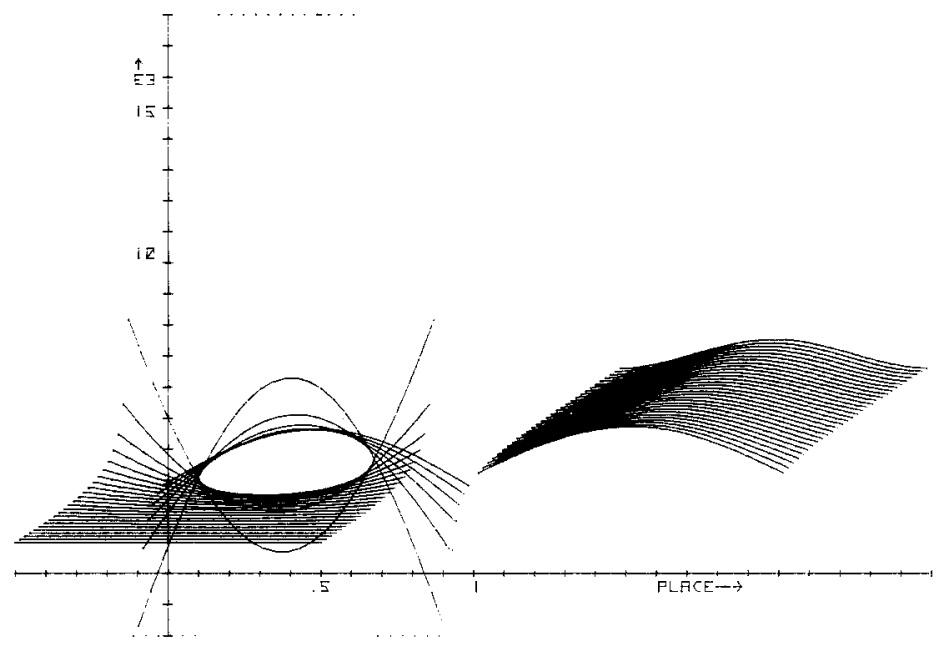

Fig. 7 Plot of $E_{3}$ (freq, place) parallel poled bar resonator.

at the central region of the bar (below precursory resonance) or at the ends of the bar (above). This inversion, or generally the dependence of $E_{3}$ on position is the main reason for considering the voltage difference $\phi$ across the electrodes as an externally controlled parameter, contrary to the resonator with field perpendicular to length where the electric field is considered as such. Figure 8 shows a plot of the local coupling factor as a function of the position in the bar, and of frequency. $k_{\text {loc }}$

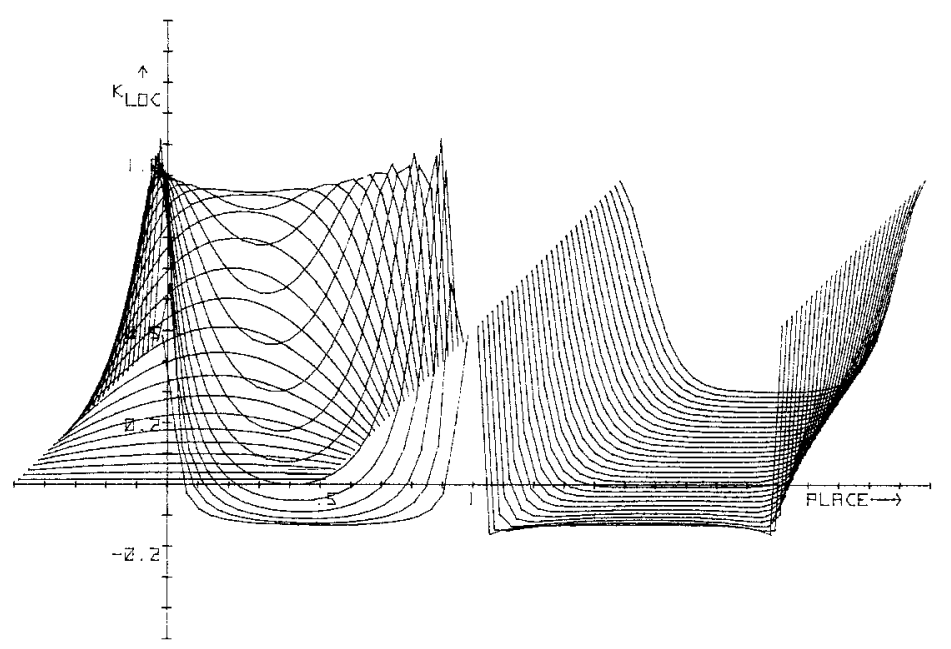

Fig. 8 Plot of $k_{\text {loc }}$ (freq, place) parallel poled resonator. 
grows rapidly in the centre until it reaches its maximum value $\left(\kappa_{33}\right)$ long before precursory resonance, where the excitation vector is then locally a median vector. With still increasing frequency, the maximum splits up into two parts, each of which shifts to the ends of the bar. In the regions where $E_{3}$ becomes negative, while $T_{3}$ still remains positive, the local coupling factor becomes negative, a phenomenon which is observed even before precursory resonance. The dramatic changes in $E_{3}$ and $T_{3}$ at this point are not found in $k_{\text {loc }}$, because the poles in $E_{s}$ and $T_{3}$ cancel each other out as a common factor in $k_{\text {loc }}$.

Though it is not clearly visible in the plot (due to large position steps in the calculation) $k_{\mathrm{loc}}$ can reach a maximum at any position in the bar at a certain frequency below resonance. The position $x_{3 m}$ where $k_{\text {loc }}$ reaches this maximum is given by

$$
x_{3 m}=\frac{1}{a} \arccos \frac{1}{\kappa_{33}} \cos \frac{a}{2} .
$$

The lowest frequency for $k_{\text {loc }}$ to reach a maximum value is found by making $x_{3 m}$ in (42) equal to zero, which yields for this frequency $a=1.59$, which is roughly at one half of the resonance frequency.

Above resonance the values of $E_{3}$ and $T_{3}$ remain more or less at the same level, and the local state vector is located close to the surface of the ellipsoid over a large part of the bar, which causes $k_{\text {loc }}$ to have a maximum value which is more or less independent of position or frequency.

\section{E. THICKNESS MODES IN THIN PLATES WITH ELECTRODED MAJOR SURFACES AND POLARIZATION PERPENDICULAR TO THESE SURFACES}

Suppose we excite an infinitely large plate with certain thickness. Then contour modes cannot arise due to the fact that the velocity of sound is finite, so no lateral motion nor lateral deformation will be present $\left(S_{1}=S_{2}=0\right)$. Again we have Maxwell's equation $\boldsymbol{\nabla} \cdot \mathbf{D}=\rho$, which yields for insulating and uncharged materials:

$$
\begin{aligned}
\frac{\partial D_{3}}{\partial x_{3}} & =0, \\
D_{1} & =0, \\
D_{2} & =0 .
\end{aligned}
$$


The condition of free major surfaces of the plate results in:

$$
T_{3}\left(x_{3}= \pm \frac{1}{2}\right)=0 \text {. }
$$

Suppose that the plate is now excited by an externally applied voltage difference:

$$
\phi\left(x_{3}=\frac{1}{2}\right)-\phi\left(x_{3}=-\frac{1}{2}\right)=\phi_{0},
$$

then this set of boundary conditions enables us to use the constituent equations in a configuration with $S$ and $D$ as independent variables:

$$
\begin{aligned}
T_{1}=T_{2} & =c_{13}^{\mathrm{D}} S_{3}-h_{31} D_{3}, \\
T_{3} & =c_{33}^{\mathrm{D}} S_{3}-h_{33} D_{3}, \\
E_{3} & =-h_{33} S_{3}+\beta_{33}^{\mathrm{S}} D_{3} .
\end{aligned}
$$

Solving these equations, together with Newton's equation, as has been outlined in the previous sections for other resonator forms, we find

$$
\begin{aligned}
T_{1}=T_{2} & =\left(\frac{c_{13}^{\mathrm{D}}}{c_{33}^{D}} h_{33} R_{\mathrm{p}}-h_{31}\right) \Phi, \\
T_{3} & =h_{33}\left(R_{p}-1\right) \Phi, \\
E_{3} & =\left(\frac{-h_{33}^{2}}{c_{33}^{D}} R_{p}+\beta_{33}^{S}\right) \Phi,
\end{aligned}
$$

where

$$
\Phi=\frac{\phi_{0} \varepsilon_{33}^{\mathrm{s}}}{\frac{h_{33}^{2}}{c_{33}^{D} \beta_{33}^{s}} \frac{2}{a} \operatorname{tg} \frac{a}{2}-1} ; \quad R_{p}=\frac{\cos a x_{3}}{\cos a / 2} .
$$

Not all these forces or fields are important, for example plots of $T_{3}$ and $E_{3}$ are trivial variations of the plots of $T_{3}$ and $E_{3}$ of the parallel poled bar and we shall not consider them here. A plot of $T_{1}$ is presented in figure 9 , which shows that even at zero frequency, $T_{1}$ has a finite value, inside the plate as well as at the surface. For increasing frequency the forces grow, even at the surface, which is a consequence of the fact that solids have a non-zero Poisson Ratio. The existence of a non-zero $T_{1}$ at $f=0$ causes a non-zero, negative coupling factor $k_{\text {loc }}$ (see figure 10) at $f=0$.

Only near resonance, does $T_{3}$ gain enough influence to render $k_{\text {loc }}$ positive. $T_{1}$ has a precursory resonance frequency of $0.885 f_{\text {res }}$ for PZT 4 as can be seen by making the denominator of $\Phi$ equal to zero. 


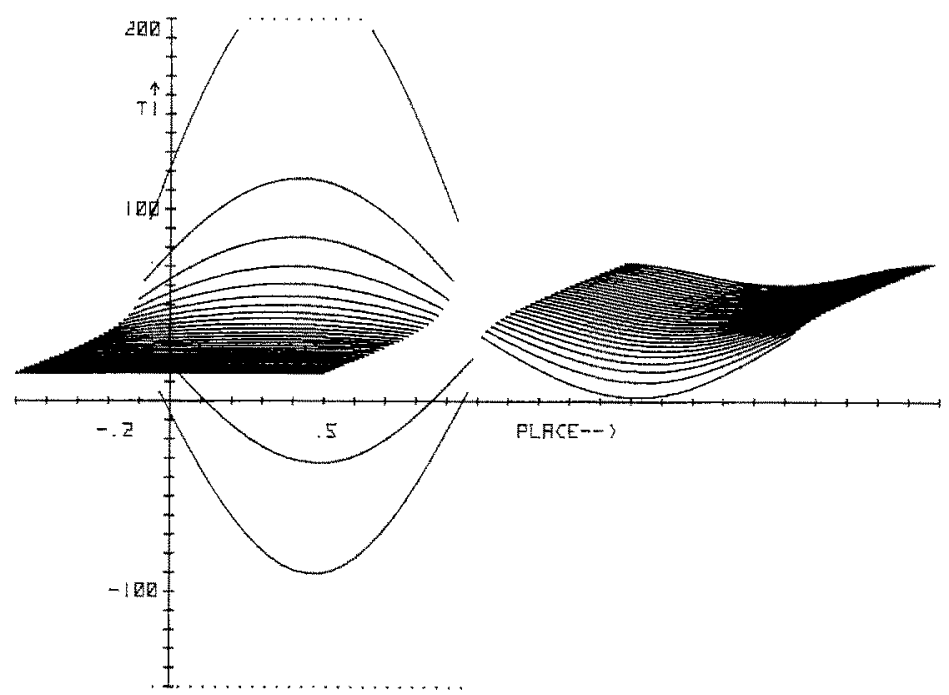

Fig. 9 Plot of $T_{1}$ (freq, place) plate resonator.

Above resonance we see almost the same deep trench as for the parallel poled bar, with two differences:

a) at the surfaces of the plate the coupling factor remains negative, while for the bar it becomes zero at the electrodes.

b) the local coupling factor does not achieve as low values for the plate as those for the bar.

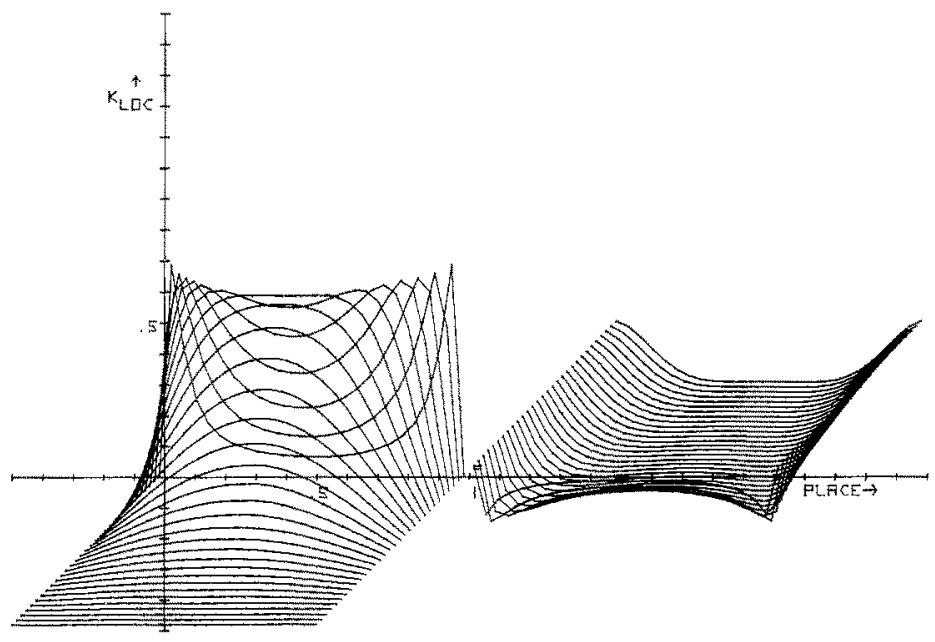

Fig. 10 Plot of $k_{\text {loc }}$ (freq, place) plate resonator. 


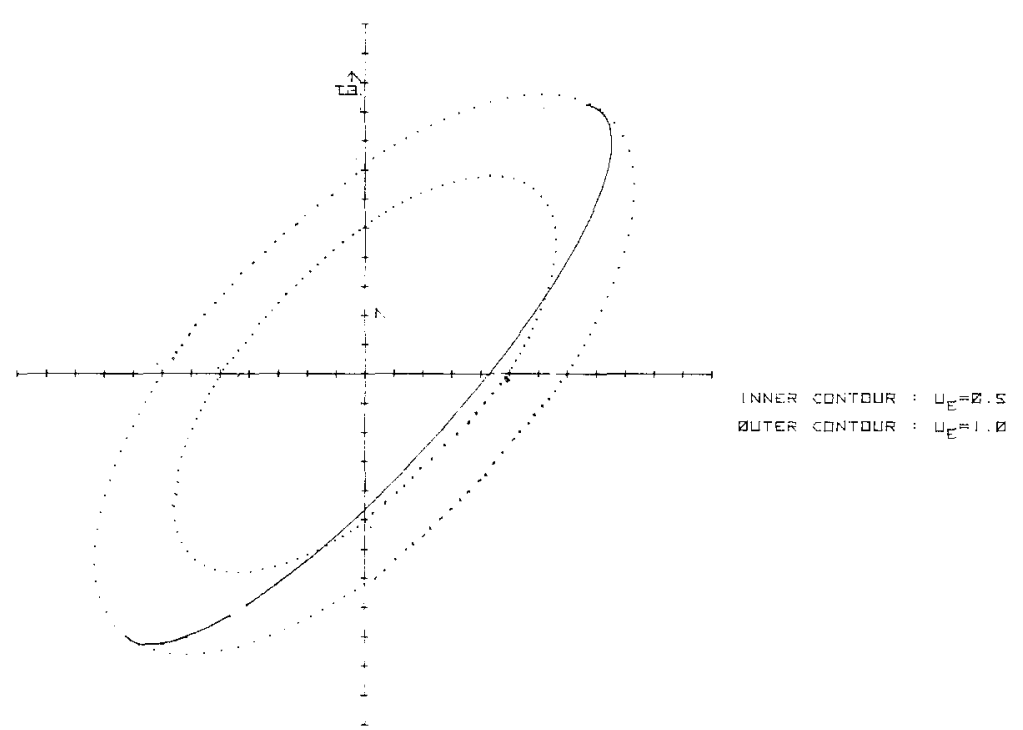

Fig. 11 Locus of $\left(T_{1}, T_{3}\right)$ plate resonator coupling state space.

We can understand this by considering figure 11 , where the locally normalized elastic part of the state vector $(E>0)$ is drawn. The locus of $T_{3}$ versus $T_{1}$ in which the frequency and place are parameters is an ellipse-like curve, of which the inclination of the long axis equals $\arctan c_{13}^{\mathrm{E}} / c_{33}^{E}$.

Here we see that the state vector below resonance becomes a median vector in the proximity of a coupling eigenstate with a coupling factor zero, whereas it again crosses the $U_{E}=\frac{1}{2}$ contour, left of the $T_{3}$ axis. Here the locus of $T_{3}$ for the bar crosses the inner contour (corresponding to the ellipsoid surface) closer to the eigenstate with $k=-k_{1}$ (see table 1); so it can be expected that $k_{\text {loc }}$ for the bar reaches lower values. The position where this locus $T_{1}-T_{3}$ for the plate crosses the $U_{E}=\frac{1}{2}$ contour above resonance moves to the right with increasing piezoelectric coefficients. It is possible for the crossing point of intersection to move to the right of the vertical axis for values of these coefficients exceeding those which are presently known, and which are not theoretically excluded. This implies that an eigenstate is almost excited in a plate vector. Until now it was accepted that it is possible to excite an eigenstate vector in a volume resonator having the shape of a short, thick cylinder in which radial resonance would 
coincide with length resonance. Such cylinders have been studied by several authors e.g. [10], and it is found that at a certain thickness/radius ratio, strong resonance is observed. It is likely that at this ratio an eigenstate is excited, which gives rise to strong coupling over a large area in the body of the resonator. We now find, under certain conditions, that it is also possible to excite an eigenstate in a plate resonator.

The total coupling factor is shown in figure 12 , where we see that the tendency of shifting to lower frequencies both for the peak and the zero crossing (if the piezoconstant is high enough) is met here just as it was for the length expander bar with field parallel. The difference between the bar and plate resonators, namely that the former had a zero approaching positive coupling factor for zero approaching frequency, while the latter then had a coupling factor of a certain negative value, is somewhat artificial, because it is induced by boundary conditions $(43,44,45)$ which explicitly exclude the phenomenon of resonating contour modes.

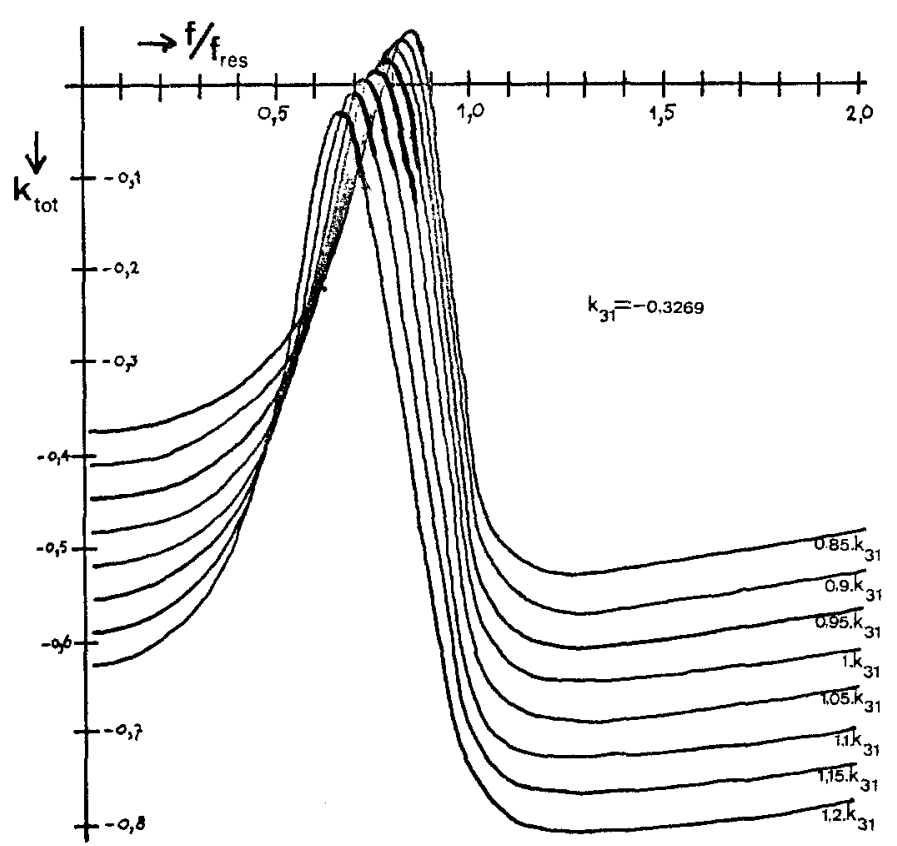

Fig. 12 Total coupling factor of a plate resonator for different values of the piezoelectric coefficient $d$. 
In the plate resonator the maximum value of $k_{\text {tot }}$ is positive, only if the piezoelectric effect is small, while for increasing piezoeffect the peak value decreases to a negative value. The reason for the negative coupling factor in strongly piezoelectric materials is that with increasing piezoeffect a larger part of the ellipse-like locus is below the "dead" mode in the negative coupling factor area. Thus a larger part of the volume of the resonator has a negative coupling because with increasing piezo-effect, the ellipse-like curve bends outwards and more volume elements in the body of the resonator move into the negative coupling area.

\section{Representation of resonators in the loss eigenstate space}

The loss eigenstates span the loss eigenspace. Any resonator with a known state vector can be represented in the loss space. The direction in which the state vector points is the same in coupling space as in loss space, as it depends only on the ratio between the entries of the vector. However the lengths are different due to different normalizations in coupling and loss spaces. In the coupling space the vectors are normalized so that

$$
U_{E}+U_{D}=1
$$

while in the loss space the vectors are normalized to have

$$
U_{E}+2 U_{M}+U_{D}=1 \text {. }
$$

It is clear that this normalization mismatch is readily overcome by renormalizing the coupling factor:

$$
k_{\text {renorm }}=\frac{2 U_{M}}{U_{E}+U_{D}+2 U_{M}} .
$$

This renormalized coupling factor now has the physical meaning of a coupled fraction. The renormalized eigencoupling factors, called eigencoupling fractions are

$$
\begin{aligned}
& f_{1}=\frac{\left(\varepsilon_{33}^{T}-\varepsilon_{33}^{S}\right)^{1 / 2}}{\left(\varepsilon_{33}^{T}\right)^{1 / 2}+\left(\varepsilon_{33}^{T}-\varepsilon_{33}^{S}\right)^{1 / 2}}, \\
& f_{2}=\frac{\left(\varepsilon_{11}^{T}-\varepsilon_{11}^{S}\right)^{1 / 2}}{\left(\varepsilon_{11}^{T}\right)^{1 / 2}+\left(\varepsilon_{11}^{T}-\varepsilon_{11}^{S}\right)^{1 / 2}}, \\
& f_{3}=0 .
\end{aligned}
$$


The corresponding eigenstates $F^{\prime}$ of $f$ are found by multiplying the eigenstates $F$ of $k$ by $1 /(1+k)^{1 / 2}$

$$
\mathbf{F}_{i}^{\prime}=\frac{\mathbf{F}_{i}}{\sqrt{1+k_{i}}}
$$

Now the eigenstates of the coupled fraction and of the loss are identically normalized and a vector can be transferred from one space to the other without alterations. Therefore, it is possible using a certain resonator geometry and known material coefficients to predict simultaneously the coupling and loss behavior of the resonator.

\section{Conclusions}

If all material coefficients of a certain material are known we can draw a spatial diagram with lines of constant coupling factor and constant loss factor. If a designer wants to realize a piezoelectric device in which he requires a certain amount of coupling, and in which he can tolerate a certain loss, he can consult this materials diagram and determine in which spatial directions he should excite the device, to obtain the desired coupling factor. In general he will find a conical surface in which all state vectors yield the same coupling factor. This conical surface intersects the ellipsoid with an elastic energy density of $\frac{1}{2}$, and this intersecting line itself will also be intersected by lines of constant loss. If there is an interval on the intersecting line in which the losses are satisfying the conditions required, this material can be used. If this is not the case, another material, with better combinations of loss and coupling must be chosen.

Received 18 December 1978

In final form 22 February 1979

\section{References}

1. Landolt-Börnstein: Elastic, piezoelectric, piezo-optic and electro-optic constants of crystals Vol 1 and Vol 2. Berlin, Springer Verlag, 1966.

2. Gruver, RM: State of the art. Review of ferroelectric ceramic materials, Tech. Rep. A FML-TR-66-164.

3. Petsch K: Ber Deuts Keram Gesell 47 (1970) 681.

4. Jaffe B, WR Cook, H Jaffé: Piezoelectric ceramics. London, Academic Press, 1971.

5. Holland R, EP EerNisse: Design of resonant piezoelectric devices. M.I.T. Research Monograph No. 56, Cambridge, Mass., M.I.T. Press, 1969. 
6. Holland R: Private communication, 1973.

7. Holland R: IEEE Trans Sonics Ultrasonics SU-15 (1968) 97.

8. All values of material coefficients apply to PZT4, and are taken from data from Berlincourt DA, DR Curran, $\mathrm{H}$ Jaffe: Piezoelectric and piezomagnetic materials. In: Physical acoustics, Vol I, part A, WP Mason, (ed), New York, Academic Press, 1964.

9. Tiersten HF: Ultrasonics, January 1970.

10. Ikegami S, I Ueda, S Kobayashi: J Acoust Soc Am 55 (1974) 331.

11. Standards on piezoelectric crystals, 1949. Proc IRE Dec. 1949, p 1378. 